\title{
GEM-type Detectors Using LIGA and Etchable Glass Technologies
}

\author{
S.K. Ahn ${ }^{1,2}$, J.G. Kim ${ }^{1,3}$, V. Perez-Mendez ${ }^{1}$, S. Chang ${ }^{1,2}$, K.H. Jackson ${ }^{4}$, J.A. Kadyk ${ }^{1}$, W.A. Wenzel ${ }^{1}$ and G. Cho $^{2}$ \\ ${ }^{1}$ Physics Div., Lawrence Berkeley National Laboratory (LBNL), Berkeley, CA 94720 \\ ${ }^{2}$ Dept. of Nuclear Eng., Korea Advanced Institute of Science and Technology, Taejon 305-701, Korea \\ ${ }^{3}$ Dept. of Physics, Myongji University, Yongin 449-728, Korea \\ ${ }^{4}$ Material Science Div., Lawrence Berkeley National Laboratory, Berkeley, CA 94720
}

\begin{abstract}
Gas electron multipliers (GEMS) have been made by a deep X-ray lithography technique (LIGA process) using synchrotron radiation on polymethylmethacrylate (PMMA) and by UV processes using a UV etchable glass. Gain, stability and rate capability for these detectors are described.

The LIGA detectors described consist of PMMA sheets of various thicknesses, $125 \mu \mathrm{m}$ to $350 \mu \mathrm{m}$, and have $150 \mu \mathrm{m} \times 150 \mu \mathrm{m}$ square holes spaced with a pitch of $300 \mu \mathrm{m}$. Thin copper electrodes are plated on the top and bottom surfaces using a Damascene method, followed by electroless plating of the copper onto a palladium-tin base layer. For various thicknesses of PMMA measurements have been made of absolute gain vs. voltage, time stability of gain, and rate capability. The operating gas mixture was usually $\mathrm{Ar} / \mathrm{CO}_{2}$ (70/30) gas, but some tests were also done using P10 gas. We also made GEM-like detectors using the UV etchable glass called Foturan, patterned by exposure to UV light and subsequent etching. A few measurements using these detectors will be reported, including avalanche gain and time stability.
\end{abstract}

\section{INTRODUCTION}

SINCE the group of Sauli introduced in 1996 the gas

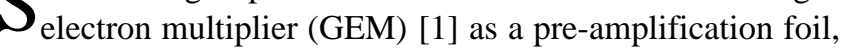
there has been a considerable effort devoted to the investigation of its characteristics, and to the improvement of its performance. Other methods of fabrication have been investigated, including dry etching and laser drilling [2].

We have made GEM-like detectors [3] by the LIGA process [4], using X-rays from an electron synchrotron (the ALS) for exposing the PMMA, and by exposure of Foturan glass [5,6] and subsequent etching. In this paper, we describe fabrication techniques and a new method for placing copper electrodes on the top and bottom GEM surfaces. We also present new measurements of GEM-like detectors made by the LIGA process, including absolute gain, time stability and rate capability, and preliminary results from the Foturan detectors.

This work was supported by the Director, Office of the Energy Research of the U.S. Department of Energy under Contract No. DE-AC03-76SF00098

\section{TECHNICAL DESCRIPTION}

We have previously described detectors [3] made by the LIGA method [4], in which low-energy X-rays are used to expose patterns on polymethylmethacrylate (PMMA) sheets. Our LIGA-fabricated detectors described here consist of thin PMMA sheets $(125 \mu \mathrm{m}-350 \mu \mathrm{m}$ thickness $)$ with arrays of $150 \mu \mathrm{m} \times 150 \mu \mathrm{m}$ square holes having steep wall sides and a pitch of $300 \mu \mathrm{m}$. These patterns are made on PMMA sheets exposed to X-rays of about $10 \mathrm{keV}$ energy through patterned gold masks. GEM-like detectors have also been made using Foturan glass of $300 \mu \mathrm{m}$ thickness. These have arrays of $130 \mu \mathrm{m} \times 130 \mu \mathrm{m}$ square holes, and also have steep wall sides and a pitch of $250 \mu \mathrm{m}$. The cross sectional dimensions of the detector sensitive region are approximately $30 \mathrm{~mm} \times 30 \mathrm{~mm}$ for the PMMA and $10 \mathrm{~mm} \times 10 \mathrm{~mm}$ for the Foturan.

\section{A. Fabrication of LIGA Detectors}

The fabrication process begins with the creation of a chromium-on-quartz photomask using an electron beam Nanowriter. The photomask is then used as a template to generate a LIGA mask: a $20 \mu \mathrm{m}$ thick gold pattern on a silicon wafer using photolithography of a spin-cast photoresist layer. The function of the LIGA mask is to produce a high differential absorption ratio at X-ray wavelengths. The two major performance considerations in masking are to ensure the proper exposure ratio between the absorbing and the transmitting regions of the mask, and profile dimensional accuracy. The LIGA mask is used as a pattern for X-ray exposure of a PMMA wafer, which will become the GEMlike device after development and plating. The X-ray source used for LIGA exposures is a beam line at the Advanced Light Source (ALS) at the Lawrence Berkeley National Laboratory (LBNL). During exposure, X-ray radiation performs chain scission on the long-chain molecules of PMMA. This effectively reduces the molecular weight in the exposed regions from $\sim 10^{6}$ to $\sim 10^{3}$ AMU's. The PMMA is then exposed to a developer (a mixture of 2-2 butoxyethoxyethanol, morpholine, 2-aminoethanol and deionized water), which selectively dissolves the lower 
molecular weight material. The unexposed PMMA remains and defines the GEM grid.

\section{B. Fabrication of Foturan Detectors}

The Institute of Microtechnology in Mainz (IMM), Germany [5], has developed a photo-etchable glass, with the trade name Foturan, which is manufactured by the Schott glass Co. [6]. It is an alkali-alumosilicate-glass, whose photosensitive characteristics arise from additions of $\mathrm{Ce}_{2} \mathrm{O}_{3}$ and $\mathrm{Ag}_{2} \mathrm{O}$. This photosensitivity allows it to be structured by UV photolithography for a variety of purposes. Foturan has mechanical, thermal and electrical properties similar to conventional glass, and has a bulk resistivity $\rho=8.1 \times 10^{12} \Omega \mathrm{cm}$ at $25^{\circ} \mathrm{C}$. For our Foturan detectors, we used a mask of $20 \mu \mathrm{m}-$ thick nickel mesh with $125 \mu \mathrm{m} \times 125 \mu \mathrm{m}$ square holes and a pitch of $250 \mu \mathrm{m}$. The exposure was made with light of $300 \mathrm{~nm}$ wavelength. The exposed glass is etched using 10\% hydrofluoric acid; the etching rate of exposed regions is about 10-15 times faster than that of the unexposed regions. The sides of holes etched into the material are nearly perpendicular to the surfaces. After this process of etching, the final hole size is $130 \mu \mathrm{m} \times 130 \mu \mathrm{m}$ and the thickness is $300 \mu \mathrm{m}$. We contracted with Mikroglas in Mainz, Germany [7] to do the lithography and etching that produced the detectors reported in this paper. The microscopic picture of patterned Foturan wafer is shown in Fig. 1.

\section{Electrodes Plating on Detectors}

For both these fabrication techniques, we deposited copper plate electrodes on the top and bottom surfaces of the detectors. The electrodes have a thickness of $1 \mu \mathrm{m}$, and are made in the following way. The surface plating begins with a step known as the Damascene method [8]: this prevents copper being plated on the walls of the holes. At first, an anthracene solution in ethyl alcohol is used to cover both surfaces of the device as well as filling in the volume of the holes. Then the dried anthracene is removed from the top and bottom surfaces, but still remains in the holes. Next, a thin coating of palladium-tin [9], which provides an initiator for the copper plate, is deposited on the top and bottom surfaces of the device. Next, the anthracene is removed from the holes by ultrasonic agitation in an alcohol solution, and copper is electroless-plated [9] onto the palladium-tin layer, to the desired $1 \mu \mathrm{m}$ thickness.

\section{Experimental Setup}

The detector was placed between two electrode planes, the drift (cathode) and collection planes (anode), which were spaced by $3.2 \mathrm{~mm}$ and $1.2 \mathrm{~mm}$ (or no spacing), respectively from the wafer (see Fig. 2). The drift plane was a thin stainless steel wire grid. A copper plated ceramic layer was used as the collection plane, and was connected to a pulse height analyzer through a calibrated amplifier. In this study, the drift field was fixed at $1.2 \mathrm{kV} / \mathrm{cm}$, or at $40 \mathrm{~V} / \mathrm{cm}$ for some tests. The applied voltage on the detectors is defined as the voltage difference between the top and bottom electrodes. In order to provide high voltage on detectors safely with no spark damage, a network of voltage dividers and protective series resistances was used, as described in detail in [10]. As is well known, some of the electrons from the GEM avalanche are lost during the collection step by going to the bottom electrode of detector rather than to the collection plane [11]. So we investigated this effect by setting the collection gap to zero in several measurements. This also helped to avoid using the complex voltage-dividing network, which can be a source of noise.

The gas mixture used was either $\mathrm{Ar} / \mathrm{CO}_{2}$ (70/30), or $\mathrm{P} 10$, $\mathrm{Ar} / \mathrm{CH}_{4}(90 / 10)$, and the source of primary ionization was either an ${ }^{55} \mathrm{Fe}(5.9 \mathrm{keV})$ source $(\sim 40 \mu \mathrm{Ci})$ or vanadium-filtered $\mathrm{X}$-rays from an X-ray tube having a copper target, and operated with an anode voltage of $6 \mathrm{kV}$.

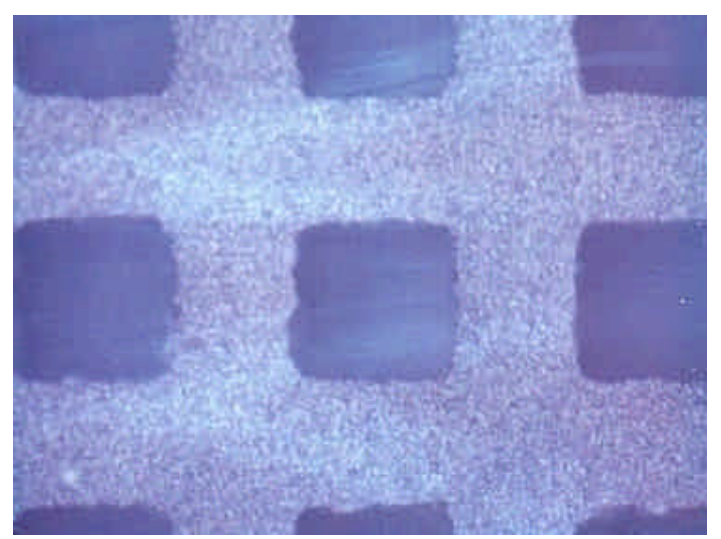

Fig. 1. Picture of the hole pattern produced with UV exposure and etching of a $300 \mu \mathrm{m}$-thick Foturan wafer. The hole size is $130 \mu \mathrm{m} \times 130 \mu \mathrm{m}$ and the pitch is $250 \mu \mathrm{m}$.

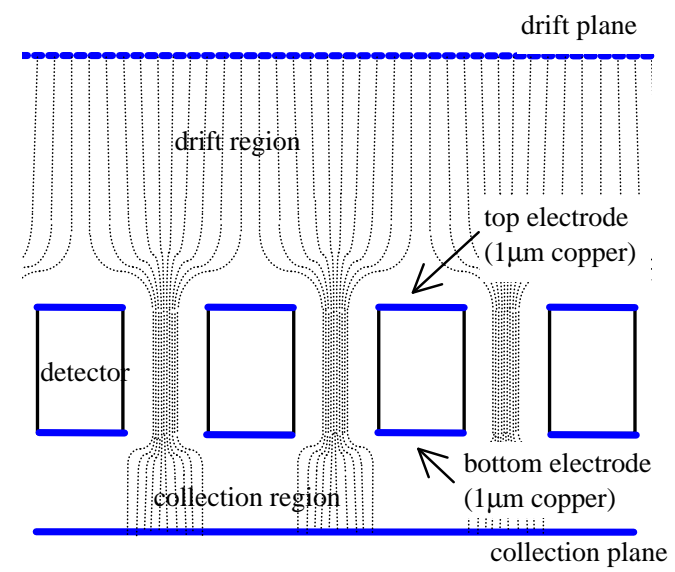

Fig. 2. Schematic representation of the structure of the GEM-type detector, coupled with drift and collection planes. The drift gap was $3.2 \mathrm{~mm}$ and the collection gap was either $1.2 \mathrm{~mm}$ or zero. 


\section{RESULTS AND DISCUSSION}

We give below the results of a number of detectors made by the LIGA and etchable glass techniques. The leakage resistance of each detector was determined by measuring the current as a function of applied voltage between the top and bottom conducting surfaces. This resistance is more than $10^{14} \Omega$ for the LIGA detectors, and about $10^{11} \Omega$ for the Foturan glass detectors. The volume resistivities specified by the manufacturers are $2-14 \times 10^{15} \Omega \mathrm{cm}$ and $8.1 \times 10^{12} \Omega \mathrm{cm}$, for the PMMA and Foturan glass, respectively.

\section{A. Results from LIGA Detectors}

Fig. 3 shows gain for several LIGA detectors with different thickness. The maximum gains were limited by electrical micro-discharges on the devices, when fluctuations occurred in the collected currents. From the measurements of avalanche gain vs. drift field, our LIGA detectors obtain the best gain performance with a drift field of about 1.2 $1.5 \mathrm{kV} / \mathrm{cm}$. The gains were also measured in the $\mathrm{P} 10$ gas with zero collection gap, as shown in Fig. 4. In this case, however, we set the drift field at $40 \mathrm{~V} / \mathrm{cm}$ because avalanche gain was not much smaller than for higher fields, and we obtained better energy resolution. It should be noted that no attempt has been made to optimize the relative sizes of the holes, thickness, and pitch: Such optimization is expected to result in a considerable gain increase.

As shown in Fig. 5, gain stabilities for various detectors were measured in $\mathrm{Ar} / \mathrm{CO}_{2}$ (70/30) gas. We used a collimated ${ }^{55} \mathrm{Fe}$ source at a count rate of $50-100 \mathrm{~Hz} / \mathrm{mm}^{2}$ in all measurements. The gain decreased about $15 \%$ within about 1.5 hours and then stabilized for thicknesses of $125-150 \mu \mathrm{m}$, when the collection gap was zero. However, in the case of $1.2 \mathrm{~mm}$-collection gap and $5 \mathrm{kV} / \mathrm{cm}$ collection field, the gain

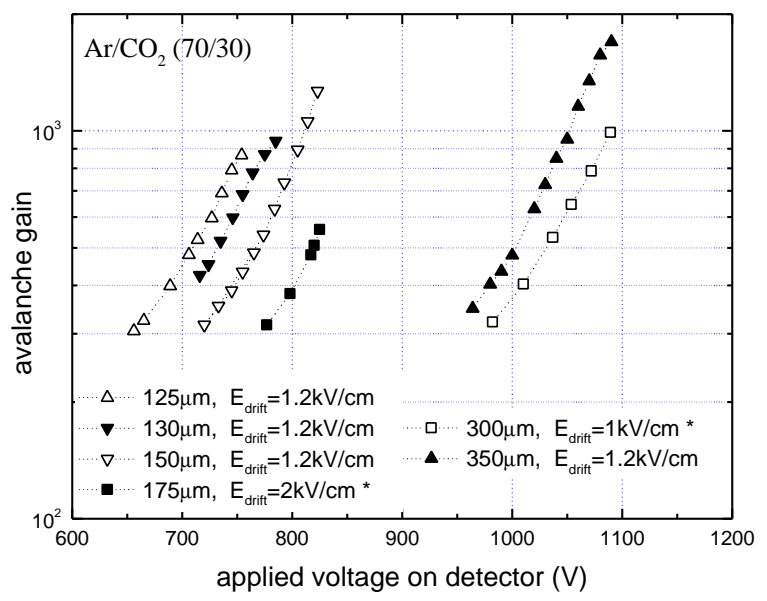

Fig. 3. Measurements of avalanche gain as a function of applied voltage across LIGA detectors of $125-350 \mu \mathrm{m}$ thickness, as indicated on the graph, in $\mathrm{Ar} / \mathrm{CO}_{2}(70 / 30)$ gas. All measurements have been performed with an ${ }^{55} \mathrm{Fe}$ source, and up to voltages at which fluctuation of current become prominent. The drift gap was $3.2 \mathrm{~mm}$, and $E_{\text {drift }}$ was $1.2 \mathrm{kV} / \mathrm{cm}$ or $2 \mathrm{kV} / \mathrm{cm}$. The collection gap was zero except for tests marked with *, where the collection gap was $1.2 \mathrm{~mm}$ and $\mathrm{E}_{\text {collect }}$ was $5 \mathrm{kV} / \mathrm{cm}$. drop was less than $10 \%$. We obtained similar results from the thicker $(300$ and $350 \mu \mathrm{m})$ detectors. We believe that because of the longer path through holes of the thicker detectors, there is increased surface charging and a large gain drop, as seen in Fig. 5.

A pulse height spectrum from an ${ }^{55} \mathrm{Fe}$ source is shown in Fig. 6. The FWHM of the principal peak $(5.9 \mathrm{keV})$ is usually about $30 \%$, and the best obtained result is $20 \%$ FWHM. We believe that the square hole geometry contributes to a somewhat poorer resolution than would be obtained with circular holes.

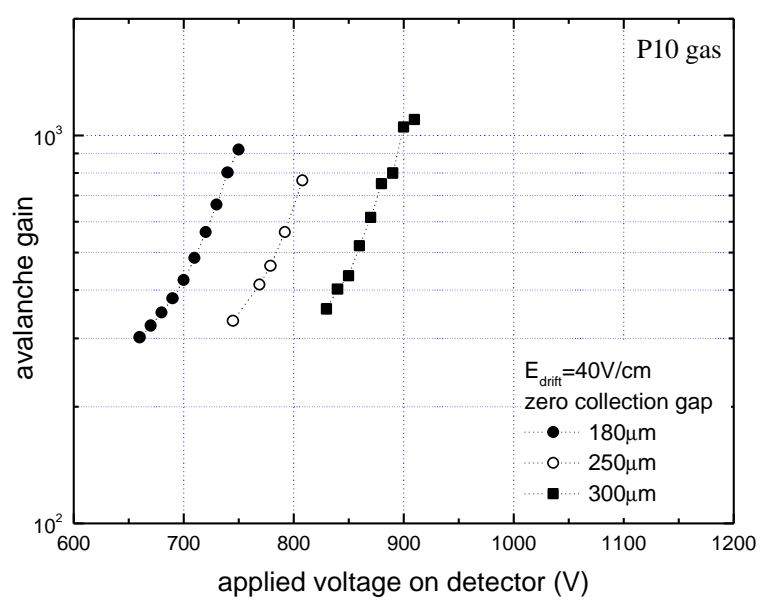

Fig. 4. Measurements of avalanche gain as a function of applied voltage across LIGA detectors of 180, 250 and $300 \mu \mathrm{m}$ thickness in P10 gas. $\mathrm{E}_{\text {drift }}=$ $40 \mathrm{~V} / \mathrm{cm}$ and the collection gap was zero.

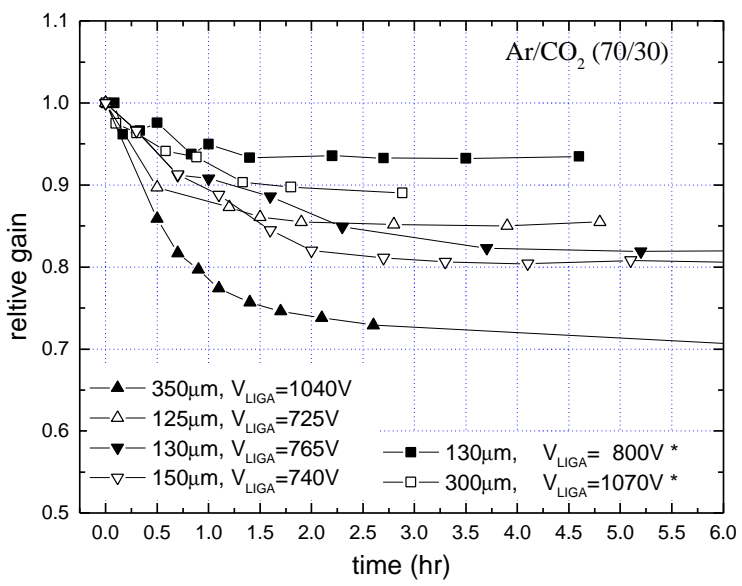

Fig. 5. Gain variation with time of several detectors of different thicknesses. For the thinner detectors, $\sim 130 \mu \mathrm{m}$ thick, there was a gain decrease of about $15 \%$ after about 1.5 hours, and then the gain stabilized. The drift gap was $3.2 \mathrm{~mm}$, and $E_{\text {drift }}$ was $1.2 \mathrm{kV} / \mathrm{cm}$. The collection gap was zero, except for those marked as $* \mathrm{E}_{\text {drift }}=2 \mathrm{kV} / \mathrm{cm}, \mathrm{E}_{\text {collect }}=5 \mathrm{kV} / \mathrm{cm}$ and the collection gap was $1.2 \mathrm{~mm}$. 


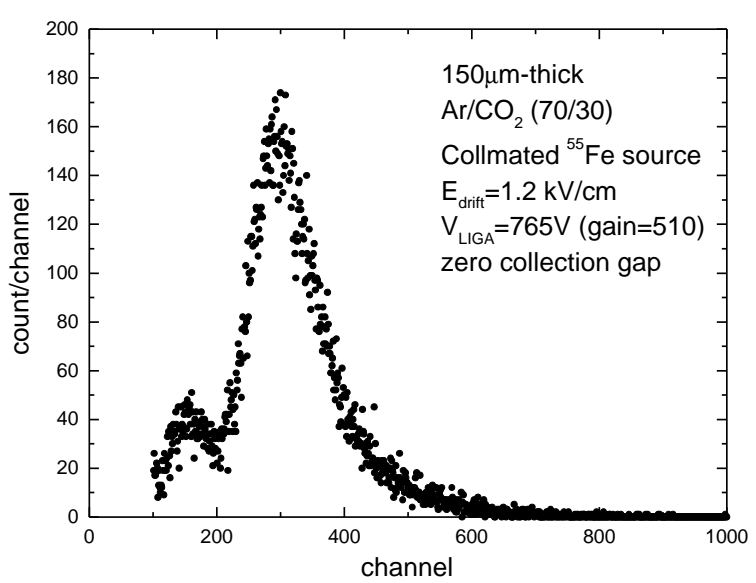

Fig. 6. Pulse height spectrum obtained using a LIGA detector of $150 \mu \mathrm{m}$ thickness and an ${ }^{55} \mathrm{Fe}$ source. The FWHM is $30 \%$ (see text).

(a)

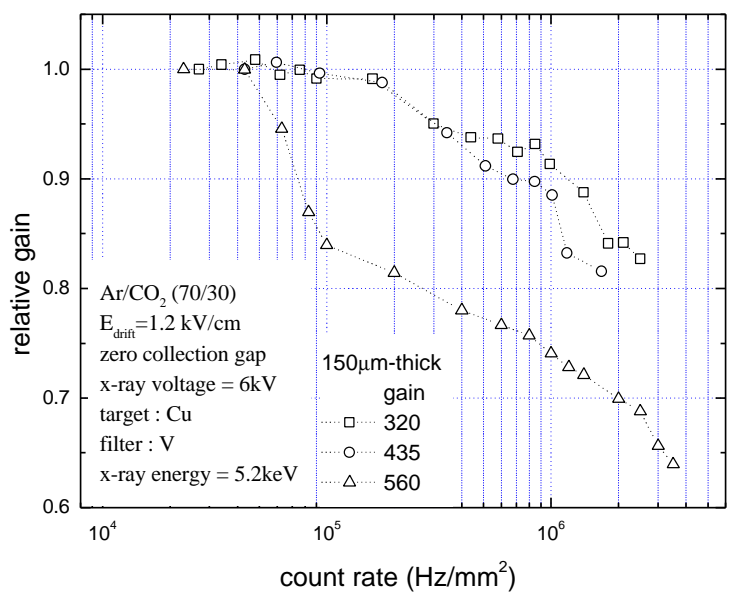

(b)

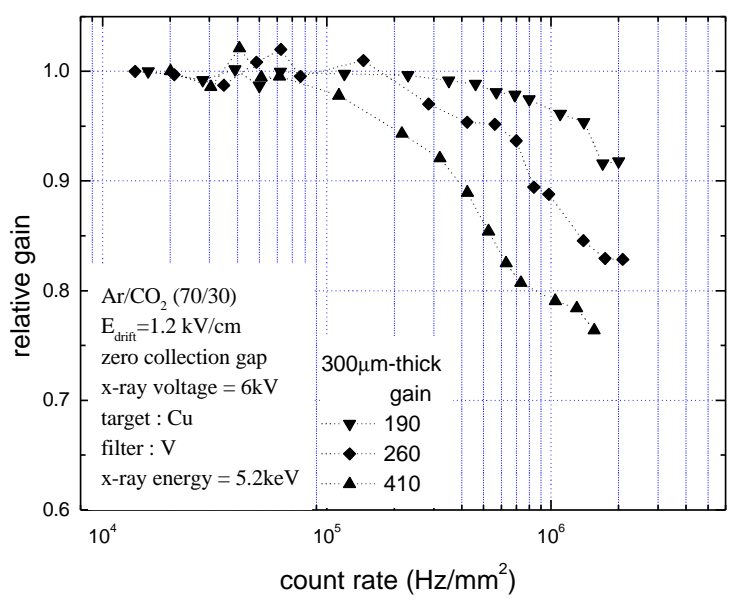

Fig. 7. Relative gain as a function of count rate for the $150 \mu \mathrm{m}$ (a) and $300 \mu \mathrm{m}$ (b) -thick LIGA detectors. X-rays of about $5.2 \mathrm{keV}$ were used as a source of primary ionization.
For future experiments, which have to work in the environment of very high luminosity, high rate detectors are needed. Using our X-ray generator, we measured the relative gain of the LIGA detectors as a function of rate for three different gains. As shown in Fig. 7, the gain decrease in detectors running at a gain of 400 occurs at counting rates exceeding about $10^{5} \mathrm{~Hz} / \mathrm{mm}^{2}$. At the higher gain of $\mathrm{G}=560$, however, the gain decrease begins at a few tens of $\mathrm{kHz} / \mathrm{mm}^{2}$. Comparing Fig. 7 (a) and (b), the rate capability is only slightly worse, if at all, for the thicker detectors, but it depends sensitively on the operating gain.

\section{B. Results from Foturan Glass Detectors}

We have measured the gain and time stability of Foturan glass detectors. Fig. 8 shows the avalanche gain for these detectors in $\mathrm{Ar} / \mathrm{CO}_{2}(70 / 30)$ gas. The maximum gains were limited by fluctuations in the collected currents. Even though the wafer thickness is $300 \mu \mathrm{m}$, we obtained avalanche gains at a smaller applied voltage than for LIGA detectors of similar thickness. This result perhaps can be partially explained by the different hole size and pitch than for the X-ray LIGA detectors. We determined the gain from the principal peak of the ${ }^{55} \mathrm{Fe}$ measured pulse height spectrum, but the energy resolution was poor, as shown in Fig. 9 For the Foturan and LIGA detectors of similar thickness $(\sim 300 \mu \mathrm{m})$, the pitch and hole size are: (pitch/hole) $=(300 / 150)_{\text {LIGA }}$ and $(250 / 130)_{\text {Foturan. }}$ A change of GEM geometry can result in very different gain properties. These results represent the first measurements of GEM-like detectors using an etchable glass. Further investigations should help determine the potential of this method as the alternative form of GEM detector fabrication. The time stability, which is shown Fig. 10, is quite similar to that of the PMMA detector made by the LIGA process.

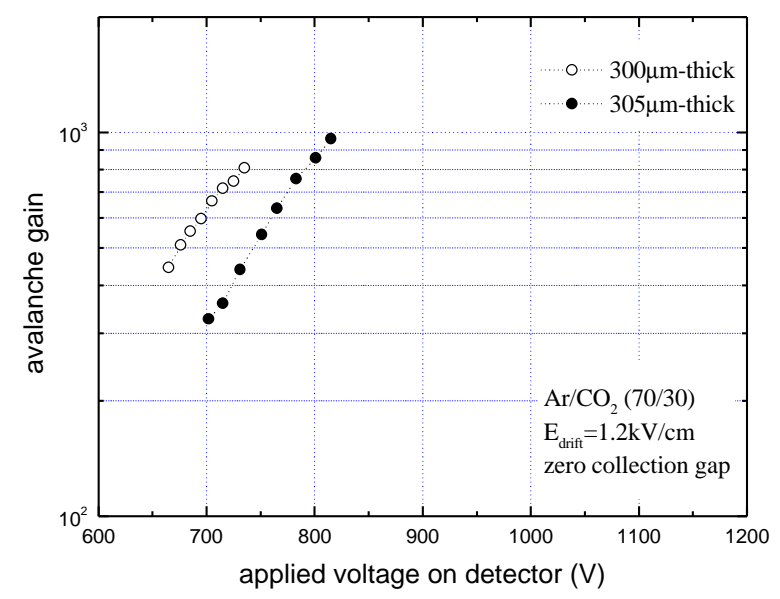

Fig. 8. Measurements of avalanche gain as a function of an applied voltage across Foturan detectors of two $300 \mu$ m thickness in $\mathrm{Ar} / \mathrm{CO}_{2}$ (70/30) gas. $\mathrm{E}_{\text {drift }}$ was $1.2 \mathrm{kV} / \mathrm{cm}$ and the collection gap was zero. 


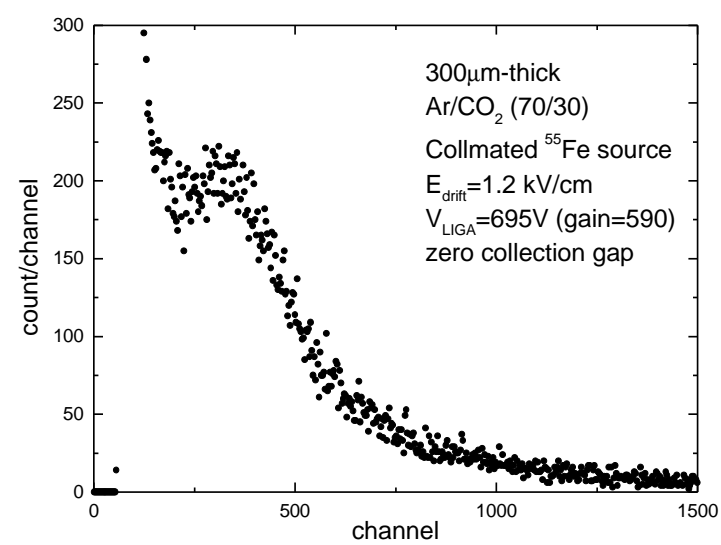

Fig. 9. Pulse height spectrum for an ${ }^{55} \mathrm{Fe}$ source obtained using a Foturan glass detector of $300 \mu \mathrm{m}$ thickness (see text).

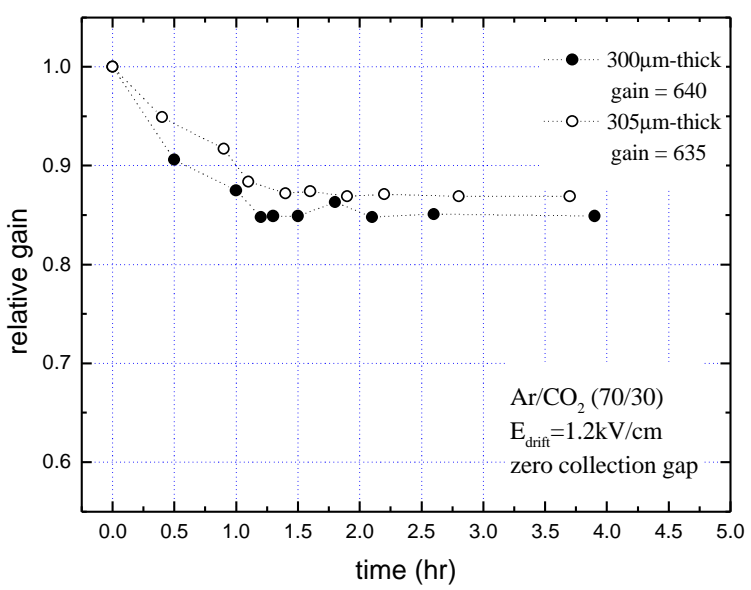

Fig. 10. Gain variation vs. time of Foturan detectors of $300 \mu \mathrm{m}$ thickness using a $40 \mu \mathrm{Ci}{ }^{55} \mathrm{Fe}$ source. About $15 \%$ decrease of gain is observed after 1.5 hours.

\section{CONCLUSION}

GEM-type detectors were made by the LIGA technique of exposing PMMA to low energy X-rays, and by UV light exposure of the etchable glass, Foturan. Both techniques are shown to make functioning GEM-type detectors. Using detectors of several thicknesses, we measured reasonable performance with regard to gain, time stability and rate capability. We also introduced a new method for placing copper electrodes on the top and bottom GEM surfaces. The results from detectors using Foturan glass indicate the possibility of using this alternative technique for GEM manufacturing. Compared with previously used techniques, the different properties of this glass substrate (e.g. thickness, robustness) might provide an extended range of application of GEM-type devices.

Another possibility under investigation is to use either of these techniques to make copper molds, by electroplating copper into the hole patterns, following the original meaning of LIGA: lithography, electroforming and molding. These copper molds could then be used for making GEM-like detectors from molded plastic wafers.

\section{ACKNOWLEDGMENT}

We would like to thank Ms. Kenney of Schott in Yonkers, NY and Ms. Vogel of Mikroglas in Mainz, Germany who constructed the Foturan glass patterns.

\section{REFERENCES}

[1] F. Sauli, "GEM: A new concept for electron amplification in gas detectors", Nucl. Instr. Meth., A386, pp. 531-534, 1997.

[2] W.K. Pitts, M.D. Martin, S. Belolipetskiy, M. Crain, J.B. Huntchins, S. Mators, K.M. Walsh and K. Solberg, "Development and operation of laser machined microwell detectors", Nucl. Instr. Meth. A438, p277, 1999.

[3] H.K. Kim, K. Jackson, W.S. Hong, I.J. Park, S.H. Han, J. Kadyk, V. Perez-Mendez, W. Wenzel and G. Cho, "Application of the LIGA process for fabrication of gas avalanche devices", IEEE Trans Nucl Sci 47 (3), 923-927, 2000.

[4] P. Rai-Choudhury, "Handbook of microlithography, micromachining, and microfabrication", vol. 2, ch. 6, SPIE, 1997.

[5] WWW: http://www.imm-mainz.de

[6] Schott Co., 3 Odell Plaza, Yonkers, NY, USA. WWW: http://Www.schottglass.com

[7] Mgt mikroglas tech., Galileo-Galilei-Str. 28, 55129 Mainz, Germany, WWW: http://www.mikroglas.de

[8] Jon Reid, Steve Mayer, Eliot Broadbent, Erich Klawuhn, Kaihan Ashtiani, Novellus Systems, "Factors Influencing Damascene Feature Fill Using Copper PVD and Electroplating", Technical Documents from Novellus System, Inc.,

WWW: http://www.novellus.com/damascus/tec/tec_20.asp

[9] Technical Information for Palladium-Tin Catalyst and LC Electroless Copper Process, M\&T Chemicals Inc., Rahway, NJ, USA.

[10] H.S. Cho, J. Kadyk, S.H. Han, W.S. Hong, V. Perez-Mendez, W. Wenzel, K. Pitts, M.D. Martin and J.B. Hutchins, "GEM: Performance and aging tests”, IEEE Trans. Nucl. Sci., vol. 46, no. 3, pp. 306-311, 1999.

[11] R. Bellazzini, A. Brez, G. Gariano, L. Latronico, N. Lumb, G. Spandre, M. M. Massai, R. Raffo, M. A. Spezziga, "What is the real gas gain of a standard GEM?”, Nucl. Instr. Meth., A419, pp429-437, 1998. 